\title{
Studies on Cognitive Enhancing Agents. II. Antiamnestic and Antihypoxic Activities of 1-Aryl-2-(2-aminoethoxy)ethanols
}

\author{
Satoshi Ono,* Tetsuo Yamafuji, Hisaaki Chaki, Hajime Morita, Yozo Todo, \\ Mutsuko MaEkawa, Kazunori Kitamura, Masaru TAI, and Hirokazu Narita
}

Research Laboratories, Toyama Chemical Co., Ltd., 2-4-1 Shimookui, Toyama 930, Japan.

Received January 5, 1995; accepted May 11, 1995

A series of 2-(2-aminoethoxy)-1-phenylethanols having a variety of $\mathrm{N}$ - and phenyl-substitution patterns as well as 5- and 6-membered heteroaryl counterparts of our prototype compound 1 (2-(2-dimethylaminoethoxy)-1phenylethanol) have been prepared and evaluated for antiamnestic and antihypoxic activities. Compound $3 \mathrm{~b}$, the 3-methylphenyl analogue of 1 , proved to be significantly more potent than 1 in reversing electroconvulsive shock-induced amnesia as well as $\mathrm{CO}_{2}$-induced learning-impairment in mice. It exhibited low acute toxicity in mice and afforded a greater brain/serum concentration ratio than 1 after oral administration to rats.

Key words 1-aryl-2-(2-aminoethoxy)ethanol; cognitive enhancing agent; antiamnestic activity; antihypoxic activity; tacrine

In the previous paper ${ }^{1)}$ describing our preliminary efforts directed to developing a new, potent agent for the treatment of the dementia observed in Alzheimer and multi-infarct diseases, we disclosed that 2-(2-dimethylaminoethoxy)-1-phenylethanol (1) displays moderate antiamnestic and antihypoxic activities in mice. Other notable pharmacological features of $\mathbf{1}$ are its low acute toxicity, comparable to indeloxazine, and good ability to permeate through the blood-brain barrier in rats. We next examined structural modifications of this prototype compound 1 as shown in Chart 3, namely, variations of the $N, N$ dimethyl group, introduction of simple substituents on the phenyl ring, and replacement of the phenyl group with some heteroaryl groups. It has been found that among the compounds prepared in this study, 2-(2-dimethylaminoethoxy)-1-(3-methylphenyl)ethanol (3b) is signif- cantly more active than 1 in amnesia-reversal as well as in protective effect against hypoxia in mice.

\section{Chemistry}

As shown in Chart 2, all of the 1-aryl-2-aminoethoxyethanols were prepared by reaction of aryloxirane with 2-aminoethanols in the presence of potassium tertbutoxide in dimethyl sulfoxide (DMSO). The intermediate oxiranes, except styrene oxide, were obtained from<smiles>CN(C)CCOCC(O)c1ccccc1</smiles>

1

Chart 1

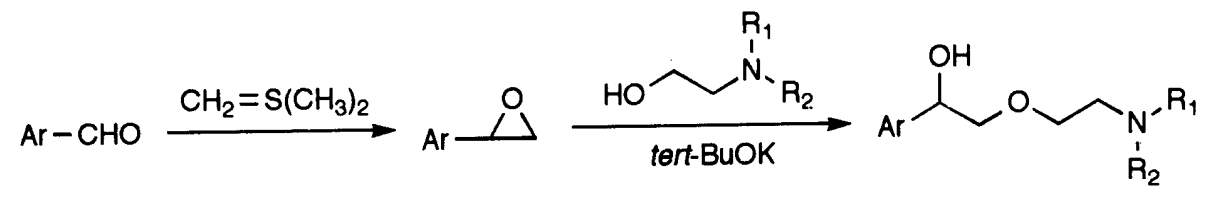

Chart 2<smiles>[2H]N([P])CCOCC(O)c1ccccc1</smiles>

\begin{tabular}{|c|c|c|}
\hline $2 a-g$ & $R_{1}$ & $\mathrm{R}_{2}$ \\
\hline a & $\mathrm{H}$ & $\mathrm{H}$ \\
\hline b & $\mathrm{CH}_{3}$ & $\mathrm{H}$ \\
\hline c & $\mathrm{CH}_{3} \mathrm{CH}_{2}$ & $\mathrm{CH}_{3} \mathrm{CH}_{2}$ \\
\hline d & iso- $\mathrm{C}_{3} \mathrm{H}_{7}$ & iso- $\mathrm{C}_{3} \mathrm{H}_{7}$ \\
\hline e & \multicolumn{2}{|c|}{$-\left(\mathrm{CH}_{2}\right)_{5}-$} \\
\hline$f$ & \multicolumn{2}{|c|}{$-\left(\mathrm{CH}_{2}\right)_{2}-\mathrm{O}-\left(\mathrm{CH}_{2}\right)_{2}-$} \\
\hline $\mathbf{g}$ & \multicolumn{2}{|c|}{$\begin{array}{c}-\left(\mathrm{CH}_{2}\right)_{2}-\mathrm{N}-\left(\mathrm{CH}_{2}\right)_{2}- \\
\mathrm{CH}_{3}\end{array}$} \\
\hline
\end{tabular}<smiles>CN(C)CCOCC(O)c1ccccc1</smiles>

\begin{tabular}{clll} 
3a-q & \multicolumn{2}{c}{$\mathbf{R}$} & \\
\hline a & $2-\mathrm{CH}_{3}$ & j & $4-\mathrm{F}$ \\
b & $3-\mathrm{CH}_{3}$ & k & $2,4-\left(-\mathrm{Cl}_{2}\right)$ \\
c & $4-\mathrm{CH}_{3}$ & I & $2-\mathrm{C}_{6} \mathrm{H}_{5}$ \\
d & $3-\mathrm{C}_{2} \mathrm{H}_{5}$ & m & $3-\mathrm{C}_{6} \mathrm{H}_{5}$ \\
e & $4-\mathrm{C}_{2} \mathrm{H}_{5}$ & $\mathbf{n}$ & $4-\mathrm{C}_{6} \mathrm{H}_{5}$ \\
f & $3,4-\left(\mathrm{CH}_{3}\right)_{2}$ & 0 & $3-\mathrm{OCH}_{3}$ \\
g & $2-\mathrm{Cl}$ & p & $4-\mathrm{OCH}_{3}$ \\
h & $3-\mathrm{Cl}$ & q & $4-\mathrm{OC}_{6} \mathrm{H}_{5}$ \\
i & $4-\mathrm{Cl}$ & &
\end{tabular}

Chart 3<smiles></smiles><smiles>CN(C)CCOCC(O)c1ccccc1</smiles>

\begin{tabular}{cc} 
5a-c & Aryl \\
\hline a & 2-pyridyl \\
b & 3-pyridyl \\
c & 4-pyridyl
\end{tabular}


the corresponding arylcarboxaldehydes by a methylene transfer reaction with dimethylsulfonium methylid. ${ }^{2)}$ Characterization data of new compounds are recorded in Table 3 in the experimental section.

\section{Pharmacological Results and Discussion}

All of the compounds prepared in this study were screened for antiamnestic (AA) and antihypoxic (AH) activities in mice, by observation of reversal effects on electroconvulsive shock-induced learning impairment and measurement of the survival times from hypoxia, respectively. Results are recorded in Table 1.

Most of the 2-(2-aminoethoxy)-1-phenylethanols (2ag, 3a-q) showed AH activity, although their activity levels are strongly dependent on the nature of the $\mathrm{N}$-substituent, as well as phenyl-ring substitution. However, none of the heteroaryl counterparts of our prototype compound 1 $(4 a, b, 5 a-c)$ showed AH activity, though they exhibited moderate AA activity.

Among the $\mathbf{N}$-substitution variants of our prototype 1 (compounds $2 \mathbf{a}-\mathbf{g}$ ), only the piperidine compound $2 \mathbf{e}$ shows good $\mathrm{AA}$ and $\mathrm{AH}$ activities comparable to those of 1. AA activity is not seen with the $N, N$-diethyl (2c), $N, N$-diisopropyl (2d), and $N$-methyl piperazine (2g) compounds, implying that AA activity in this series (unsubstituted phenyl) is very sensitive to the size and polarity of the $\mathrm{N}$-groups. Similar sensitivity to minor structural change can also be seen in alkyl substitution on the benzene ring. Thus, compound $\mathbf{3 b}$ having a methyl group at the 3-position displays remarkable $\mathbf{A A}$ and $\mathbf{A H}$ activities exceeding those of 1 , but interestingly, its one-carbon homologue 3d (3-ethyl) does not show AA activity. Furthermore, essentially no AA activity is seen with regioisomers of $3 \mathrm{~b}$ or with the 3,4-dimethyl derivative (3).

Introduction of a chlorine atom at the benzene ring of 1 increases AH activity significantly, as seen with $3 g-\mathbf{i}$, but causes some decrease in AA activity. On the other hand, the 4-fluoro and 2,4-dichloro compounds $(3 \mathbf{j}, \mathbf{k})$ proved much less potent than the monochloro compounds in both $\mathrm{AA}$ and $\mathrm{AH}$ activities. The activity profile of the phenyl-substitution compounds $(31-n)$ is quite dependent on the position of the phenyl group. Only the 3-phenyl compound ( $3 \mathrm{~m}$ ) shows dual activity, but its AA activity is unacceptably low. Attachment of ether functions such as a methoxy or phenoxy group on the benzene ring caused complete loss of either AA or AH activity, as seen from the data on $30-q$.

Compound $3 \mathrm{~b}$, which displayed the best activity profiles in the preliminary bioassays, was next investigated for its reversing activity on $\mathrm{CO}_{2}$-induced learning impairment in mice. Table 2 shows minimum effective doses (MEDs) for 3b and prototype 1, together with MED data for two reference drugs: tacrine ${ }^{3)}$ (a cholinesterase inhibitor) and indeloxazine $^{4)}$ (a cerebral metabolism enhancer). The impairment-reversal activity of $3 \mathrm{~b}$ was found to be almost equivalent to that of tacrine and some ten times greater than those of 1 and indeloxazine. Other notable features of $\mathbf{3 b}$ (Table 2) are: 1) $\mathbf{3 b}$ is considerably less toxic than tacrine (acute $\mathrm{LD}_{50}$ in mice: $68 \mathrm{mg} / \mathrm{kg}$ for tacrine $v$. $>500 \mathrm{mg} / \mathrm{kg}$ for $3 \mathrm{~b}$ ); 2) the brain-to-serum concentration<smiles>Cc1cccc(C(O)COCCN(C)C)c1</smiles>

3b

Chart 4

Table 1. Antiamnestic and Antihypoxic Activities of 2-(2-Aminoethoxy)1-phenylethanols and Their Heteroaryl Analogues in Mice

\begin{tabular}{|c|c|c|}
\hline Compound & $\begin{array}{c}\text { Antiamnestic activity } \\
\text { (3 mg/kg i.p. dosing) }\end{array}$ & $\begin{array}{l}\left.\text { Antihypoxic activity }{ }^{b}\right) \\
\text { (100 mg/kg p.o. dosing) }\end{array}$ \\
\hline 1 & ++ & ++ \\
\hline $2 a$ & ++ & + \\
\hline $2 \mathbf{b}$ & + & - \\
\hline $2 c$ & - & + \\
\hline $2 d$ & - & + \\
\hline $2 e$ & ++ & ++ \\
\hline $2 f$ & + & + \\
\hline $2 \mathrm{~g}$ & - & + \\
\hline $3 a$ & - & +++ \\
\hline $\mathbf{3 b}$ & +++ & +++ \\
\hline $3 c$ & + & ++ \\
\hline 3d & - & + \\
\hline $3 e$ & - & + \\
\hline $3 f$ & - & ++ \\
\hline 3g & + & $+t+$ \\
\hline 3h & + & ++++ \\
\hline $3 i$ & + & ++++ \\
\hline $3 \mathbf{j}$ & - & ++ \\
\hline 3k & - & + \\
\hline 31 & ++ & - \\
\hline $3 \mathrm{~m}$ & + & +++ \\
\hline $3 \mathbf{n}$ & - & ++++ \\
\hline 30 & + & - \\
\hline $3 p$ & ++ & - \\
\hline $3 q$ & - & +++ \\
\hline $4 \mathrm{a}$ & + & - \\
\hline $4 b$ & + & - \\
\hline $5 \mathfrak{a}$ & + & - \\
\hline 5b & ++ & - \\
\hline $5 c$ & + & - \\
\hline
\end{tabular}

a) Determined by testing reversal of electroconvulsive shock-induced amnesia. Symbols represent mean latency:,$-<60 \mathrm{~s} ;+, 60-100 \mathrm{~s} ;++, 101-150 \mathrm{~s} ;+++$, $151-300$ s. b) Determined with hypoxia models. Symbols represent \% increase in survival time against untreated animals:,$-<25 \% ;+, 25-50 \% ;++, 51-$ $75 \%$;,$++ 76-100 \%$;,$++++>100 \%$.

Table 2. Learning-Ameliorating Activity, Acute Toxicity, and Brainto-Serum Ratio of 2-(2-Dimethylaminoethoxy)-1-(3-methylphenyl)ethanol (3b) in Oral Administration

\begin{tabular}{cccc}
\hline \hline Compound & $\begin{array}{c}\text { Reversal of learning } \\
\text { impairment in mice } \\
\text { MED }^{\text {b) }}(\mathrm{mg} / \mathbf{k g})\end{array}$ & $\begin{array}{c}\text { Acute toxicity } \\
\text { in mice) } \\
\text { LD }_{\text {so }}(\mathrm{mg} / \mathbf{k g})\end{array}$ & $\begin{array}{c}\text { Brain/serum } \\
\text { ratio in rats }\end{array}$ \\
\hline 1 & 30 & $>500$ & 4.96 \\
3b & 3 & $>500$ & 8.32 \\
Tacrine & 1 & 68 & 8.47 \\
Indeloxazine & $>30$ & $444^{\text {e) }}$ & 18.46 \\
\hline
\end{tabular}

a) Amnesia was induced by exposure to $\mathrm{CO}_{2}$ immediately after the acquisition trial. b) Minimum effective dose. c) $\mathrm{LD}_{\mathrm{so}}$ were calculated from lethality during $7 \mathrm{~d}$ after dosing. d) Rats were killed $30 \mathrm{~min}$ after administration of test compounds $(30 \mathrm{mg} / \mathrm{kg})$. e) Ref. 7 .

ratio of $\mathbf{3 b}$ in rats is almost equal to that of the much more toxic tacrine.

In conclusion, the present investigation, aimed at im- 
Table 3. Characterization Data for $2 a-g, 3 a-q, 4 a, b, 5 a-c$

\begin{tabular}{|c|c|c|c|c|c|c|c|}
\hline \multirow{2}{*}{ Compd. } & \multirow{2}{*}{$\begin{array}{l}\text { Yield }^{a)} \\
(\%)\end{array}$} & \multirow{2}{*}{ Formula } & \multirow{2}{*}{$\begin{array}{c}\operatorname{mp}\left({ }^{\circ} \mathrm{C}\right) \\
\text { (Solvent for } \\
\text { crystallization) }\end{array}$} & \multicolumn{3}{|c|}{$\begin{array}{l}\text { Combustion analysis } \\
\text { Calcd (Found) (\%) }\end{array}$} & \multirow{2}{*}{${ }^{1} \mathrm{H}$-NMR spectral data $\delta$ in $\mathrm{D}_{2} \mathrm{O}$ solvent, $J$ in $\mathrm{Hz}$} \\
\hline & & & & C & $\mathbf{H}$ & $\mathbf{N}$ & \\
\hline 2a & 17 & $\begin{array}{l}\mathrm{C}_{10} \mathrm{H}_{15} \mathrm{NO}_{2} \\
(0.5 \text { fumaric acid }) \\
\cdot 0.35 \mathrm{H}_{2} \mathrm{O}\end{array}$ & $\begin{array}{l}181-182.5 \\
\left(\mathrm{IPA}-\mathrm{H}_{2} \mathrm{O}\right)\end{array}$ & $\begin{array}{r}58.69 \\
(58.75\end{array}$ & $\begin{array}{l}7.23 \\
6.97\end{array}$ & $\begin{array}{l}5.70 \\
5.59)\end{array}$ & $\begin{array}{l}3.1-3.4(2 \mathrm{H}, \mathrm{m}), 3.6-3.9(4 \mathrm{H}, \mathrm{m}), 4.97(1 \mathrm{H}, \mathrm{t}, J=6) \\
6.51(1 \mathrm{H}, \mathrm{s}), 7.44(5 \mathrm{H}, \mathrm{s})\end{array}$ \\
\hline $\mathbf{2 b}$ & 27 & $\mathrm{C}_{11} \mathrm{H}_{17} \mathrm{NO}_{2} \cdot \mathrm{HCl}$ & $\begin{array}{l}152-153 \\
(\mathrm{EtOH})\end{array}$ & $\begin{array}{r}57.01 \\
(56.80\end{array}$ & $\begin{array}{l}7.83 \\
7.99\end{array}$ & $\begin{array}{l}6.04 \\
5.75)\end{array}$ & $\begin{array}{l}2.72(3 \mathrm{H}, \mathrm{s}), 3.1-3.4(2 \mathrm{H}, \mathrm{m}), 3.7-3.9(4 \mathrm{H}, \mathrm{m}), 4.95 \\
(1 \mathrm{H}, \mathrm{t}, J=5), 7.44(5 \mathrm{H}, \mathrm{s})\end{array}$ \\
\hline $2 c^{c)}$ & 45 & $\mathrm{C}_{14} \mathrm{H}_{23} \mathrm{NO}_{2} \cdot 0.5 \mathrm{NDS}^{d}$ & $\begin{array}{c}151-153 \\
\left(\mathrm{EtOH}-\mathrm{Me}_{2} \mathrm{CO}\right)\end{array}$ & $\begin{array}{r}59.82 \\
(59.69\end{array}$ & $\begin{array}{l}7.13 \\
7.10\end{array}$ & $\begin{array}{l}3.67 \\
3.56)\end{array}$ & $\begin{array}{l}1.15(6 \mathrm{H}, \mathrm{t}, J=7.5), 2.8-3.3(6 \mathrm{H}, \mathrm{m}), 3.5-3.8(4 \mathrm{H}, \\
\mathrm{m}), 4.88(1 \mathrm{H}, \mathrm{t}, J=6), 7.39(5 \mathrm{H}, \mathrm{s}), 7.70(1 \mathrm{H}, \mathrm{dd} \\
J=8.5,7), 8.21(1 \mathrm{H}, \mathrm{d}, J=7), 8.88(1 \mathrm{H}, \mathrm{d}, J=8.5)\end{array}$ \\
\hline 2d & 6 & $\mathrm{C}_{16} \mathrm{H}_{27} \mathrm{NO}_{2} \cdot \mathrm{HCl}$ & $\begin{array}{r}116.5-118 \\
\left(\mathrm{Me}_{2} \mathrm{CO}\right)\end{array}$ & $\begin{array}{r}63.66 \\
(63.45\end{array}$ & $\begin{array}{l}9.35 \\
9.63\end{array}$ & $\begin{array}{l}4.64 \\
4.55)\end{array}$ & $\begin{array}{l}1.31(12 \mathrm{H}, \mathrm{d}, J=7), 3.1-3.4(2 \mathrm{H}, \mathrm{m}), 3.4-3.9(6 \mathrm{H}, \\
\mathrm{m}), 4.95(1 \mathrm{H}, \mathrm{t}, J=5.5), 7.44(5 \mathrm{H}, \mathrm{s})\end{array}$ \\
\hline $\mathbf{2 e}$ & 11 & $\mathrm{C}_{15} \mathrm{H}_{23} \mathrm{NO}_{2} \cdot \mathrm{HCl}$ & $\begin{array}{c}163.5-164.5 \\
\left(\text { EtOH-Et }{ }_{2} \mathrm{O}\right) \\
\text { (Ref. } 9163-165)\end{array}$ & $\begin{array}{r}63.04 \\
(62.78\end{array}$ & $\begin{array}{l}8.46 \\
8.30\end{array}$ & $\begin{array}{l}4.90 \\
4.81)\end{array}$ & $\begin{array}{l}1.4-2.0(6 \mathrm{H}, \mathrm{m}), 3.0-3.5(6 \mathrm{H}, \mathrm{m}), 3.5-4.0(4 \mathrm{H}, \mathrm{m}) \\
4.95(1 \mathrm{H}, \mathrm{t}, J=6), 7.44(5 \mathrm{H}, \mathrm{s})\end{array}$ \\
\hline $2 f$ & 16 & $\mathrm{C}_{14} \mathrm{H}_{21} \mathrm{NO}_{3} \cdot \mathrm{HCl}$ & $\begin{array}{c}153-154.5 \\
(\text { EtOH-Et } \\
\text { (Ref. } 9155-157)\end{array}$ & $\begin{array}{r}58.43 \\
(58.48\end{array}$ & $\begin{array}{l}7.71 \\
7.42\end{array}$ & $\begin{array}{l}4.87 \\
5.05)\end{array}$ & $\begin{array}{l}3.1-3.5(6 \mathrm{H}, \mathrm{m}), 3.7-4.1(8 \mathrm{H}, \mathrm{m}), 4.97(1 \mathrm{H}, \mathrm{t}, J=6) \\
7.44(5 \mathrm{H}, \mathrm{s})\end{array}$ \\
\hline $2 \mathrm{~g}$ & 12 & $\mathrm{C}_{15} \mathrm{H}_{24} \mathrm{~N}_{2} \mathrm{O}_{2} \cdot 2 \mathrm{HCl}$ & $\left(\mathrm{EtOH}-\mathrm{CH}_{2} \mathrm{Cl}_{2}\right)$ & $\begin{array}{r}53.42 \\
(53.07\end{array}$ & $\begin{array}{l}7.77 \\
7.90\end{array}$ & $\begin{array}{l}8.31 \\
8.10)\end{array}$ & $\begin{array}{l}3.04(3 \mathrm{H}, \mathrm{s}), 3.4-4.1(14 \mathrm{H}, \mathrm{m}), 4.98(1 \mathrm{H}, \mathrm{t}, J=5.5) \\
7.46(5 \mathrm{H}, \mathrm{s})\end{array}$ \\
\hline $3 \mathbf{a}$ & 11 & $\mathrm{C}_{13} \mathrm{H}_{21} \mathrm{NO}_{2} \cdot \mathrm{HCl}$ & $\begin{array}{c}198-199 \\
\text { (EtOH) }\end{array}$ & $\begin{array}{r}60.11 \\
(60.07\end{array}$ & $\begin{array}{l}8.54 \\
8.83\end{array}$ & $\begin{array}{l}5.39 \\
5.44)\end{array}$ & $\begin{array}{l}2.37(3 \mathrm{H}, \mathrm{s}), 2.29(6 \mathrm{H}, \mathrm{s}), 3.3-3.6(2 \mathrm{H}, \mathrm{m}), 3.7-4.1 \\
(4 \mathrm{H}, \mathrm{m}), 5.23(1 \mathrm{H}, \mathrm{t}, J=6), 7.4-7.8(4 \mathrm{H}, \mathrm{m})\end{array}$ \\
\hline 3b & 40 & $\mathrm{C}_{13} \mathrm{H}_{21} \mathrm{NO}_{2} \cdot \mathrm{HCl}$ & $\begin{array}{c}168-169 \\
\text { (IPA) }\end{array}$ & $\begin{array}{r}60.11 \\
(59.83\end{array}$ & $\begin{array}{l}8.54 \\
8.80\end{array}$ & $\begin{array}{l}5.39 \\
5.38)\end{array}$ & $\begin{array}{l}2.36(3 \mathrm{H}, \mathrm{s}), 2.88(6 \mathrm{H}, \mathrm{s}), 3.3-3.5(2 \mathrm{H}, \mathrm{m}), 3.7-4.0 \\
(4 \mathrm{H}, \mathrm{m}), 4.93(1 \mathrm{H}, \mathrm{t}, J=6), 7.3-7.5(4 \mathrm{H}, \mathrm{m})\end{array}$ \\
\hline $3 \mathbf{c}$ & 17 & $\mathrm{C}_{13} \mathrm{H}_{21} \mathrm{NO}_{2} \cdot \mathrm{HCl}$ & $\begin{array}{c}181.5-183 \\
(\text { EtOH })\end{array}$ & $\begin{array}{r}60.11 \\
(60.01\end{array}$ & $\begin{array}{l}8.54 \\
8.52\end{array}$ & $\begin{array}{l}5.39 \\
5.54)\end{array}$ & $\begin{array}{l}2.36(3 \mathrm{H}, \mathrm{s}), 2.95(6 \mathrm{H}, \mathrm{s}), 3.3-3.6(2 \mathrm{H}, \mathrm{m}), 3.7-4.1 \\
(4 \mathrm{H}, \mathrm{m}), 5.07(1 \mathrm{H}, \mathrm{t}, J=6), 7.3-7.7(4 \mathrm{H}, \mathrm{m})\end{array}$ \\
\hline 3d & 16 & $\mathrm{C}_{14} \mathrm{H}_{23} \mathrm{NO}_{2} \cdot \mathrm{HCl}$ & $\begin{array}{c}159.5-160.5 \\
\text { (EtOH-AcOEt) }\end{array}$ & $\begin{array}{r}61.41 \\
(61.52\end{array}$ & $\begin{array}{l}8.44 \\
8.99\end{array}$ & $\begin{array}{l}5.12 \\
5.33)\end{array}$ & $\begin{array}{l}1.21(3 \mathrm{H}, \mathrm{t}, J=7.5), 2.68(2 \mathrm{H}, \mathrm{q}, J=7.5), 2.87(6 \mathrm{H}, \mathrm{s}) \\
3.2-3.5(2 \mathrm{H}, \mathrm{m}), 3.5-4.0(4 \mathrm{H}, \mathrm{m}), 4.97(1 \mathrm{H}, \mathrm{t}, J=6)\end{array}$ \\
\hline 3e & 13 & $\mathrm{C}_{14} \mathrm{H}_{23} \mathrm{NO}_{2} \cdot \mathrm{HCl}$ & $\begin{array}{l}201.5-203 \\
\text { (IPA) }\end{array}$ & $\begin{array}{r}61.41 \\
(61.26\end{array}$ & $\begin{array}{l}8.84 \\
9.08\end{array}$ & $\begin{array}{l}5.12 \\
5.13)\end{array}$ & $\begin{array}{l}1.19(3 \mathrm{H}, \mathrm{t}, J=7), 2.68(2 \mathrm{H}, \mathrm{q}, J=7), 2.85(6 \mathrm{H}, \mathrm{s}), \\
3.2-3.5(2 \mathrm{H}, \mathrm{m}), 3.7-4.0(4 \mathrm{H}, \mathrm{m}), 4.94(1 \mathrm{H}, \mathrm{t}, J=6) \text {, } \\
7.35(4 \mathrm{H}, \mathrm{s})\end{array}$ \\
\hline 3f & 10 & $\mathrm{C}_{14} \mathrm{H}_{23} \mathrm{NO}_{2} \cdot \mathrm{HCl}$ & $\begin{array}{l}184-185 \\
(\text { EtOH })\end{array}$ & $\begin{array}{r}61.41 \\
(61.43\end{array}$ & $\begin{array}{l}8.84 \\
9.00\end{array}$ & $\begin{array}{l}5.12 \\
5.30)\end{array}$ & $\begin{array}{l}2.27(6 \mathrm{H}, \mathrm{s}), 2.87(6 \mathrm{H}, \mathrm{s}), 3.2-3.5(2 \mathrm{H}, \mathrm{m}), 3.7-4.0 \\
(4 \mathrm{H}, \mathrm{m}), 4.90(1 \mathrm{H}, \mathrm{t}, J=5.5), 7.20(3 \mathrm{H}, \mathrm{s})\end{array}$ \\
\hline 3g & 11 & $\begin{array}{l}\mathrm{C}_{12} \mathrm{H}_{18} \mathrm{NO}_{2} \mathrm{Cl} \cdot \mathrm{HCl} \\
\cdot 0.2 \mathrm{H}_{2} \mathrm{O}\end{array}$ & $\begin{array}{c}203-204 \\
(\text { EtOH) }\end{array}$ & $\begin{array}{r}50.79 \\
(50.82\end{array}$ & $\begin{array}{l}6.89 \\
6.91\end{array}$ & $\begin{array}{l}4.94 \\
4.78)\end{array}$ & $\begin{array}{l}2.92(6 \mathrm{H}, \mathrm{s}), 3.3-3.5(2 \mathrm{H}, \mathrm{m}), 3.7-4.1(4 \mathrm{H}, \mathrm{m}), 5.39 \\
(1 \mathrm{H}, \mathrm{dd}, J=6,5), 7.4-7.8(4 \mathrm{H}, \mathrm{m})\end{array}$ \\
\hline $3 \mathbf{h}$ & 10 & $\mathrm{C}_{12} \mathrm{H}_{18} \mathrm{NO}_{2} \mathrm{Cl} \cdot \mathrm{HCl}$ & $\begin{array}{l}170-171 \\
\left(\mathrm{Me}_{2} \mathrm{CO}\right)\end{array}$ & $\begin{array}{r}51.44 \\
(51.37\end{array}$ & $\begin{array}{l}6.84 \\
7.05\end{array}$ & $\begin{array}{l}5.00 \\
4.73)\end{array}$ & $\begin{array}{l}2.89(6 \mathrm{H}, \mathrm{s}), 3.3-3.5(2 \mathrm{H}, \mathrm{m}), 3.7-4.2(4 \mathrm{H}, \mathrm{m}), 4.97 \\
(1 \mathrm{H}, \mathrm{t}, J=5), 7.4-7.7(4 \mathrm{H}, \mathrm{m})\end{array}$ \\
\hline $\mathbf{3 i}$ & 14 & $\begin{array}{l}\mathrm{C}_{12} \mathrm{H}_{18} \mathrm{NO}_{2} \mathrm{Cl} \cdot \mathrm{HCl} \\
\cdot 0.125 \mathrm{H}_{2} \mathrm{O}\end{array}$ & $\begin{array}{l}188.5-190 \\
\left(\mathrm{EtOH}-\mathrm{Et}_{2} \mathrm{O}\right)\end{array}$ & $\begin{array}{r}51.03 \\
(51.27\end{array}$ & $\begin{array}{l}6.87 \\
6.99\end{array}$ & $\begin{array}{l}4.96 \\
4.66)\end{array}$ & $\begin{array}{l}2.86(6 \mathrm{H}, \mathrm{s}), 3.2-3.5(2 \mathrm{H}, \mathrm{m}), 3.6-4.0(4 \mathrm{H}, \mathrm{m}), 4.95 \\
(1 \mathrm{H}, \mathrm{t}, J=5.5), 7.44(4 \mathrm{H}, \mathrm{s})\end{array}$ \\
\hline $\mathbf{3 j}$ & 16 & $\mathrm{C}_{12} \mathrm{H}_{18} \mathrm{NO}_{2} \mathrm{~F} \cdot \mathrm{HCl}$ & $\begin{array}{l}171-172 \\
\text { (EtOH) }\end{array}$ & $\begin{array}{r}54.65 \\
(54.41\end{array}$ & $\begin{array}{l}7.26 \\
7.38\end{array}$ & $\begin{array}{l}5.31 \\
5.30)\end{array}$ & $\begin{array}{l}2.88(6 \mathrm{H}, \mathrm{s}), 3.3-3.5(2 \mathrm{H}, \mathrm{m}), 3.7-4.0(4 \mathrm{H}, \mathrm{m}), 4.97 \\
(1 \mathrm{H}, \mathrm{t}, J=6), 7.0-7.6(4 \mathrm{H}, \mathrm{m})\end{array}$ \\
\hline 3k & 14 & $\mathrm{C}_{12} \mathrm{H}_{17} \mathrm{NO}_{2} \mathrm{Cl}_{2} \cdot \mathrm{HCl}$ & $\begin{array}{l}184-184.5 \\
\text { (EtOH) }\end{array}$ & $\begin{array}{r}45.80 \\
(45.59\end{array}$ & $\begin{array}{l}5.77 \\
5.68\end{array}$ & $\begin{array}{l}4.45 \\
4.48)\end{array}$ & $\begin{array}{l}2.90(6 \mathrm{H}, \mathrm{s}), 3.2-3.5(2 \mathrm{H}, \mathrm{m}), 3.6-4.0(4 \mathrm{H}, \mathrm{m}), 5.33 \\
(1 \mathrm{H}, \mathrm{dd}, J=6.5,5), 7.2-7.7(3 \mathrm{H}, \mathrm{m})\end{array}$ \\
\hline $\mathbf{3}$ & 19 & $\mathrm{C}_{18} \mathrm{H}_{23} \mathrm{NO}_{2} \cdot \mathrm{HCl}$ & $\begin{array}{l}217.5-218.5 \\
(\text { EtOH })\end{array}$ & $\begin{array}{r}67.17 \\
(67.16\end{array}$ & $\begin{array}{l}7.52 \\
7.51\end{array}$ & $\begin{array}{l}4.35 \\
4.75)\end{array}$ & $\begin{array}{l}3.02(6 \mathrm{H}, \mathrm{s}), 3.3-3.5(2 \mathrm{H}, \mathrm{m}), 3.6-3.9(4 \mathrm{H}, \mathrm{m}), 5.02 \\
(1 \mathrm{H}, \mathrm{dd}, J=7.5,4.5), 7.1-7.9(3 \mathrm{H}, \mathrm{m})\end{array}$ \\
\hline $3 m$ & 15 & $\begin{array}{l}\mathrm{C}_{18} \mathrm{H}_{23} \mathrm{NO}_{2} \cdot \mathrm{HCl} \\
\cdot 0.3 \mathrm{H}_{2} \mathrm{O}\end{array}$ & $\begin{array}{c}138-140 \\
\left(\mathrm{EtOH}-\mathrm{Et}_{2} \mathrm{O}\right)\end{array}$ & $\begin{array}{r}66.06 \\
(66.05\end{array}$ & $\begin{array}{l}7.58 \\
7.48\end{array}$ & $\begin{array}{l}4.28 \\
4.22)\end{array}$ & $\begin{array}{l}2.91(6 \mathrm{H}, \mathrm{s}), 3.2-3.5(2 \mathrm{H}, \mathrm{m}), 5.09(1 \mathrm{H}, \mathrm{t}, J=5.5) \\
7.30(9 \mathrm{H}, \mathrm{m})\end{array}$ \\
\hline $3 n$ & 35 & $\mathrm{C}_{18} \mathrm{H}_{23} \mathrm{NO}_{2} \cdot \mathrm{HCl}$ & $\begin{array}{l}217-218.5 \\
\text { (EtOH) }\end{array}$ & $\begin{array}{r}67.17 \\
(67.16\end{array}$ & $\begin{array}{l}7.52 \\
7.51\end{array}$ & $\begin{array}{l}4.35 \\
4.75)\end{array}$ & $\begin{array}{l}2.86(6 \mathrm{H}, \mathrm{s}), 3.2-3.5(2 \mathrm{H}, \mathrm{m}), 3.7-4.1(4 \mathrm{H}, \mathrm{m}), 5.02 \\
(1 \mathrm{H}, \mathrm{t}, J=6), 7.4-7.9(9 \mathrm{H}, \mathrm{m})\end{array}$ \\
\hline 30 & 24 & $\mathrm{C}_{13} \mathrm{H}_{21} \mathrm{NO}_{3} \cdot \mathrm{HCl}$ & $\begin{array}{l}146-147 \\
(\mathrm{EtOH})\end{array}$ & $\begin{array}{r}56.62 \\
(56.52\end{array}$ & $\begin{array}{l}8.04 \\
8.26\end{array}$ & $\begin{array}{l}5.08 \\
4.97)\end{array}$ & $\begin{array}{l}2.92(6 \mathrm{H}, \mathrm{s}), 3.3-3.6(2 \mathrm{H}, \mathrm{m}), 3.8-4.1(4 \mathrm{H}, \mathrm{m}), 4.96 \\
(1 \mathrm{H}, \mathrm{t}, J=5.5), 7.1-7.7(4 \mathrm{H}, \mathrm{m})\end{array}$ \\
\hline $3 p$ & 6 & $\mathrm{C}_{13} \mathrm{H}_{21} \mathrm{NO}_{3} \cdot \mathrm{HCl}$ & $\begin{array}{c}171-173 \\
\text { (EtOH) }\end{array}$ & $\begin{array}{r}56.62 \\
(56.41\end{array}$ & $\begin{array}{l}8.04 \\
8.30\end{array}$ & $\begin{array}{l}5.08 \\
5.10)\end{array}$ & $\begin{array}{l}2.90(6 \mathrm{H}, \mathrm{s}), 3.3-3.5(2 \mathrm{H}, \mathrm{m}), 3.6-4.1(4 \mathrm{H}, \mathrm{m}), 4.93 \\
(1 \mathrm{H}, \mathrm{t}, J=6), 7.05(2 \mathrm{H}, \mathrm{d}, J=6), 7.38(2 \mathrm{H}, \mathrm{d}, J=9)\end{array}$ \\
\hline $3 q$ & 12 & $\mathrm{C}_{18} \mathrm{H}_{23} \mathrm{NO}_{3} \cdot \mathrm{HCl}$ & $\begin{array}{l}174.5-176.5 \\
(\mathrm{MeCN})\end{array}$ & $\begin{array}{r}63.99 \\
(63.91\end{array}$ & $\begin{array}{l}7.16 \\
7.31\end{array}$ & $\begin{array}{l}4.15 \\
4.07)\end{array}$ & $\begin{array}{l}3.01(6 \mathrm{H}, \mathrm{s}), 3.3-3.6(2 \mathrm{H}, \mathrm{m}), 3.7-4.1(4 \mathrm{H}, \mathrm{m}), 5.06 \\
(1 \mathrm{H}, \mathrm{t}, J=6), 6.9-7.7(9 \mathrm{H}, \mathrm{m})\end{array}$ \\
\hline $4 a$ & 9 & $\mathrm{C}_{10} \mathrm{H}_{17} \mathrm{NO}_{3} \cdot \mathrm{HCl}$ & $\begin{array}{l}128.5-130 \\
(\mathrm{EtOH})\end{array}$ & $\begin{array}{r}50.96 \\
(50.72\end{array}$ & $\begin{array}{l}7.70 \\
7.90\end{array}$ & $\begin{array}{l}5.94 \\
5.84)\end{array}$ & $\begin{array}{l}2.89(6 \mathrm{H}, \mathrm{s}), 3.2-3.5(2 \mathrm{H}, \mathrm{m}), 3.6-4.0(4 \mathrm{H}, \mathrm{m}), 4.93 \\
(1 \mathrm{H}, \mathrm{t}, J=6), 6.52(1 \mathrm{H}, \mathrm{s}), 7.4-7.6(2 \mathrm{H}, \mathrm{m})\end{array}$ \\
\hline $4 \mathbf{b}$ & 6 & $\mathrm{C}_{10} \mathrm{H}_{17} \mathrm{NO}_{2} \mathrm{~S} \cdot \mathrm{HCl}$ & $\begin{array}{l}165.5-166 \\
\left(\mathrm{EtOH}_{-} \mathrm{Et}_{2} \mathrm{O}\right)\end{array}$ & $\begin{array}{r}47.70 \\
(47.56\end{array}$ & $\begin{array}{l}7.21 \\
7.22\end{array}$ & $\begin{array}{l}5.56 \\
5.40)\end{array}$ & $\begin{array}{l}2.87(6 \mathrm{H}, \mathrm{s}), 3.2-3.5(2 \mathrm{H}, \mathrm{m}), 3.7-4.0(4 \mathrm{H}, \mathrm{m}), 5.05 \\
(\mathrm{HH}, \mathrm{t}, J=6), 7.1-7.3(2 \mathrm{H}, \mathrm{m}), 7.4-7.7(1 \mathrm{H}, \mathrm{m})\end{array}$ \\
\hline $5 \mathbf{a}$ & 8 & $\mathrm{C}_{1 \mathrm{1}} \mathrm{H}_{18} \mathrm{~N}_{2} \mathrm{O}_{2} \cdot 2 \mathrm{HCl}$ & $\begin{array}{c}179-179.5 \\
(\text { EtOH-AcOEt) }\end{array}$ & $\begin{array}{r}46.65 \\
(46.52\end{array}$ & $\begin{array}{l}7.12 \\
7.23\end{array}$ & $\begin{array}{l}9.89 \\
9.62)\end{array}$ & $\begin{array}{l}2.88(6 \mathrm{H}, \mathrm{s}), 3.2-3.5(2 \mathrm{H}, \mathrm{m}), 3.7-4.2(4 \mathrm{H}, \mathrm{m}), 5.44 \\
(1 \mathrm{H}, \mathrm{t}, J=4), 7.8-8.2(2 \mathrm{H}, \mathrm{m}), 8.4-8.8(2 \mathrm{H}, \mathrm{m})\end{array}$ \\
\hline $5 \mathbf{b}$ & 5 & $\begin{array}{l}\mathrm{C}_{11} \mathrm{H}_{18} \mathrm{~N}_{2} \mathrm{O}_{2} \cdot 2 \mathrm{HCl} \\
\cdot 0.2 \mathrm{H}_{2} \mathrm{O}\end{array}$ & $\begin{array}{c}143-146 \\
\text { (EtOH) }\end{array}$ & $\begin{array}{r}46.06 \\
(45.93\end{array}$ & $\begin{array}{l}7.17 \\
7.40\end{array}$ & $\begin{array}{l}9.77 \\
9.86)\end{array}$ & $\begin{array}{l}2.94(6 \mathrm{H}, \mathrm{s}), 3.3-3.6(2 \mathrm{H}, \mathrm{m}), 3.8-4.1(4 \mathrm{H}, \mathrm{m}), 5.32 \\
(1 \mathrm{H}, \mathrm{t}, J=5.5), 8.0-8.3(1 \mathrm{H}, \mathrm{m}), 8.6-9.0(3 \mathrm{H}, \mathrm{m})\end{array}$ \\
\hline sc & 7 & $\mathrm{C}_{11} \mathrm{H}_{18} \mathrm{~N}_{2} \mathrm{O}_{2} \cdot 2 \mathrm{HCl}$ & $\begin{array}{l}183.5-184.5 \\
\left(\mathrm{EtOH}-\mathrm{Et}_{2} \mathrm{O}\right)\end{array}$ & $\begin{array}{r}46.65 \\
(46.39\end{array}$ & $\begin{array}{l}7.12 \\
7.15\end{array}$ & $\begin{array}{l}9.89 \\
9.74)\end{array}$ & $\begin{array}{l}2.90(6 \mathrm{H}, \mathrm{s}), 3.2-3.5(2 \mathrm{H}, \mathrm{m}), 3.6-4.1(4 \mathrm{H}, \mathrm{m}), 5.30 \\
(1 \mathrm{H}, \mathrm{t}, J=5), 8.07(2 \mathrm{H}, \mathrm{d}, J=6.5), 8.73(2 \mathrm{H}, \mathrm{d}, J=6.5)\end{array}$ \\
\hline
\end{tabular}

a) Yield from the corresponding aldehyde. b) IPA: iso-PrOH. c) Ref. 8 recorded as free bases. d) Naphthalene-1,5-disulfonic acid. 
proving the AA and $\mathrm{AH}$ activity profile of 1 , has been rewarded with the finding of an advanced model compound $\mathbf{3 b}$, within a series of $\mathbf{N}$-group variants $(2 \mathrm{a}-\mathrm{g})$, substitution compounds on the benzene ring (3a-q), and 5- and 6-membered heteroaryl analogues $(4 a, b$, $5 a-c)$. Compound $3 b$ is significantly more potent than 1 in reversing amnesia and hypoxia in mice, while retaining the low toxicity of 1.

\section{Experimental ${ }^{5)}$}

2-[2-( $N, N$-Dimethylamino)ethoxy]-1-(3-methylphenyl)ethanol Hydrochloride (3b) (General Procedure) The intermediate (3-methylphenyl)oxirane, bp $80-85^{\circ} \mathrm{C} / 6 \mathrm{Torr}$ (ref. $654^{\circ} \mathrm{C} / 1.2 \mathrm{Torr}$ ), was obtained from 3-methylbenzaldehyde in $97 \%$ yield according to a literature procedure, except for the use of trimethylsulfonium methylsulfate/sodium methoxide in $\mathrm{CH}_{3} \mathrm{CN}$ instead of trimethylsulfonium bromide/sodium hydride in dimethyl sulfoxide (DMSO). ${ }^{1} \mathrm{H}-\mathrm{NMR}\left(\mathrm{CDCl}_{3}\right) \delta: 2.35(3 \mathrm{H}, \mathrm{s}), 2.79$ (lH, dd, $J=5.5,2.5 \mathrm{~Hz}), 3.13(1 \mathrm{H}, \mathrm{dd}, J=5.5,4 \mathrm{~Hz}), 3.83(1 \mathrm{H}, \mathrm{dd}, J=4$, $2.5 \mathrm{~Hz}), 7.0-7.5(4 \mathrm{H}, \mathrm{m})$

The oxirane obtained above $(10 \mathrm{~g}, 0.075 \mathrm{~mol})$ was added to a stirred and heated $\left(60-65^{\circ} \mathrm{C}\right)$ mixture of potassium tert-butoxide $(25 \mathrm{~g}$, $0.22 \mathrm{~mol})$ and $N, N$-dimethylethanolamine $(40 \mathrm{~g}, 0.45 \mathrm{~mol})$ in DMSO $(30 \mathrm{ml})$. After having been stirred at the same temperature for $1 \mathrm{~h}$, the reaction mixture was poured into a mixture of ice-water $(150 \mathrm{ml})$ and toluene $(150 \mathrm{ml})$. The whole was acidified to $\mathrm{pH} 1$ with $6 \mathrm{~N} \mathrm{HCl}$, and the layers were separated. The aqueous layer was basified to $\mathrm{pH} 10.5$ with $\mathrm{K}_{2} \mathrm{CO}_{3}$, then extracted with $\mathrm{CHCl}_{3}(100 \mathrm{ml} \times 2)$. The combined extracts were washed with water, dried, and concentrated. A solution of the residue in acetone $(75 \mathrm{ml})$ was treated with $6 \mathrm{~N}$ ethanolic $\mathrm{HCl}(12 \mathrm{ml}$, $0.72 \mathrm{~mol})$ at $10-20^{\circ} \mathrm{C}$ before dilution with AcOEt $(75 \mathrm{ml})$. Precipitated crystals were collected by filtration and recrystallized from iso-PrOH to give $3 \mathrm{~b}(8.7 \mathrm{~g}, 42 \%)$ as colorless needles, $\mathrm{mp} 168-169^{\circ} \mathrm{C}$.

The following compounds $(2 a-g, 3 a, c-q, 4 a, b$ and $5 a-c)$ were prepared in the same manner as described for $\mathbf{3 b}$.

Characterization data for new oxiranes prepared from the corresponding arylcarboxaldehydes are given below.

(3-Ethylphenyl)oxirane: $96 \%$ yield. bp $70-74^{\circ} \mathrm{C} / 0.7$ Torr. ${ }^{1} \mathrm{H}-\mathrm{NMR}$ $\left(\mathrm{CDCl}_{3}\right) \delta: 1.23(3 \mathrm{H}, \mathrm{t}, J=7.5 \mathrm{~Hz}), 2.4-2.9(3 \mathrm{H}, \mathrm{m}), 3.10(1 \mathrm{H}, \mathrm{dd}$, $J=5.5,4 \mathrm{~Hz}), 3.81(1 \mathrm{H}, \mathrm{dd}, J=4,2.5 \mathrm{~Hz}), 6.9-7.4(4 \mathrm{H}, \mathrm{m})$. Anal. Calcd for $\mathrm{C}_{10} \mathrm{H}_{12} \mathrm{O}$ : C, 81.04; $\mathrm{H}, 8.16$. Found: $\mathrm{C}, 80.92 ; \mathrm{H}, 8.06$.

(4-Ethylphenyl)oxirane: $97 \%$ yield. bp $77-87^{\circ} \mathrm{C} / 0.9$ Torr. ${ }^{1} \mathrm{H}-\mathrm{NMR}$ $\left(\mathrm{CDCl}_{3}\right) \delta: 1.22(3 \mathrm{H}, \mathrm{t}, J=7.5 \mathrm{~Hz}), 2.66(2 \mathrm{H}, \mathrm{q}, J=7.5 \mathrm{~Hz}), 2.81(1 \mathrm{H}$, dd, $J=5.5,2.5 \mathrm{~Hz}$ ), $3.10(1 \mathrm{H}, \mathrm{dd}, J=5.5,4 \mathrm{~Hz}), 3.81(1 \mathrm{H}, \mathrm{dd}, J=4$, $2.5 \mathrm{~Hz}), 7.18(2 \mathrm{H}, \mathrm{d}, J=15 \mathrm{~Hz}), 7.22(2 \mathrm{H}, \mathrm{d}, J=15 \mathrm{~Hz})$. Anal. Calcd for $\mathrm{C}_{10} \mathrm{H}_{12} \mathrm{O}: \mathrm{C}, 81.04 ; \mathrm{H}, 8.16$. Found: $\mathrm{C}, 80.87 ; \mathrm{H}, 8.08$.

2-Biphenyloxirane: $97 \%$ yield. bp $160-170^{\circ} \mathrm{C} / 1.5$ Torr. ${ }^{1} \mathrm{H}-\mathrm{NMR}$ $\left(\mathrm{CDCl}_{3}\right) \delta: 2.70(1 \mathrm{H}, \mathrm{dd}, J=5.5,2.5 \mathrm{~Hz}), 2.96(1 \mathrm{H}, \mathrm{dd}, J=5.5,4 \mathrm{~Hz})$, $3.78(1 \mathrm{H}, \mathrm{dd}, J=4,2.5 \mathrm{~Hz}), 7.0-7.5(9 \mathrm{H}, \mathrm{m})$. Anal. Calcd for $\mathrm{C}_{14} \mathrm{H}_{12} \mathrm{O}$ : C, 85.68; H, 6.16. Found: C, 85.54; H, 6.18 .

3-Biphenyloxirane: $95 \%$ yield. bp $120-125^{\circ} \mathrm{C} / 0.5$ Torr. ${ }^{1} \mathrm{H}-\mathrm{NMR}$ $\left(\mathrm{CDCl}_{3}\right) \delta: 2.80(1 \mathrm{H}, \mathrm{dd}, J=5.5,2.5 \mathrm{~Hz}), 3.14(1 \mathrm{H}, \mathrm{dd}, J=5.5,4 \mathrm{~Hz})$, $3.90(1 \mathrm{H}, \mathrm{dd}, J=4,2.5 \mathrm{~Hz}), 7.1-7.7(9 \mathrm{H}, \mathrm{m})$. Anal. Calcd for $\mathrm{C}_{14} \mathrm{H}_{12} \mathrm{O}$ : C, 85.68; H, 6.16. Found: C, 85.87; H, 6.27.

(4-Phenoxyphenyl)oxirane: $96 \%$ yield. bp $118-121^{\circ} \mathrm{C} / 1$ Torr. ${ }^{1} \mathrm{H}$ NMR $\left(\mathrm{CDCl}_{3}\right) \delta: 2.78(1 \mathrm{H}, \mathrm{dd}, J=5.5,2.5 \mathrm{~Hz}), 3.13(1 \mathrm{H}, \mathrm{dd}, J=5.5$, $4 \mathrm{~Hz}), 3.83(1 \mathrm{H}, \mathrm{dd}, J=4,2.5 \mathrm{~Hz}), 6.8-7.5(9 \mathrm{H}, \mathrm{m})$. Anal. Calcd for $\mathrm{C}_{14} \mathrm{H}_{12} \mathrm{O}_{2}$ : C, 79.22; H, 5.70. Found: C, 79.50; H, 5.75.

3-Furyloxirane: $85 \%$ yield. bp $62-65^{\circ} \mathrm{C} / 20$ Torr. ${ }^{1} \mathrm{H}-\mathrm{NMR}\left(\mathrm{CDCl}_{3}\right)$ $\delta: 2.85(1 \mathrm{H}, \mathrm{dd}, J=5,2.5 \mathrm{~Hz}), 3.08(1 \mathrm{H}, \mathrm{dd}, J=5,4 \mathrm{~Hz}), 3.78(1 \mathrm{H}, \mathrm{dd}$, $J=4,2.5 \mathrm{~Hz}), 6.27(1 \mathrm{H}, \mathrm{d}, J=1 \mathrm{~Hz}), 7.3-7.4(1 \mathrm{H}, \mathrm{m}), 7.4-7.5(1 \mathrm{H}$, m). MS $m / z: 110\left(\mathrm{M}^{+}\right)$.

Reversal of Electroconvulsive Shock (ECS)-Induced Impairment of a Pascive Avoidance Response Groups of 10 male ddY mice, 6-7 weeks old and weighing 26-37 grams, were used. Assay was carried out according to a reported procedure ${ }^{10}$ by using a two-compartment step-through passive avoidance apparatus consisting of an illuminated compartment $(10 \times 13 \times 15 \mathrm{~cm})$ and a darkened grid-floor-equipped compartment $(25 \times 13 \times 23 \mathrm{~cm})$ with an opening $(3 \times 4 \mathrm{~cm})$ between the boxes. The passive avoidance training was given by application of $1.5 \mathrm{~mA}$ current for $3 \mathrm{~s}$ to the grid. Each test compound dissolved in physiological saline was dosed intraperitoneally $1 \mathrm{~h}$ before the training. Immediately after the training, ECS $(20 \mathrm{~mA}, 0.5 \mathrm{~s})$ was administered to the eyes, then after $24 \mathrm{~h}$, retention of the inhibitory avoidance response was measured over a period of $300 \mathrm{~s}$.

Antihypoxic Activity Groups of 10 female ddY mice (6 weeks old) were used. Two mice were placed in a $300 \mathrm{ml}$ glass container into which a 4:96 (v/v) mixed gas of $\mathrm{O}_{2}$ and $\mathrm{N}_{2}$ was passed continuously at a flow rate of $51 / \mathrm{min}$. A test compound was orally administered $30 \mathrm{~min}$ before this treatment. Time in seconds before respiratory interception was recorded as the survival time.

Reversal of $\mathrm{CO}_{2}$-Induced Impairment of a Passive Avoidance Response Groups of 10 male ddY mice (6-7 weeks old) were used. This assay was performed according to a published procedure. ${ }^{11)} \mathrm{Im}$ mediately after being given the passive avoidance training described in the foregoing section, each mouse was brought to suspended animation by placing it in a $300 \mathrm{ml}$ glass container into which $\mathrm{CO}_{2}$ gas was passed continuously at a flow rate of $5 \mathrm{l} / \mathrm{min}$ for $30 \mathrm{~s}$. The mouse was then animated by artificial respiration, and a test compound was administered orally for evaluation of retention of the passive avoidance response. Dosages for MED determination were: $1=1.0,3.0,10$ and $30 \mathrm{mg} / \mathrm{kg}$; $3 \mathrm{~b}=1.0,3.0,10$ and $30 \mathrm{mg} / \mathrm{kg} ;$ tacrine $=0.1,0.3,1.0,3.0$, and $10 \mathrm{mg} / \mathrm{kg}$; indeloxazine $=1.0,3.0,10$, and $30 \mathrm{mg} / \mathrm{kg}$. MED as determined by the Kruskal-Wallis test followed by the Mann-Whitney $U$-test were statistically significant $(p<0.05)$.

Acknowledgment The authors are grateful to Professor E. Yoshii, Toyama Medical and Pharmaceutical University, for his help and useful suggestions in the preparation of the manuscript.

\section{References and Notes}

1) Part I: Ono S., Yamafuji T., Chaki H., Todo Y., Maekawa M., Kitamura K., Tai M., Narita H., Chem. Pharm. Bull., 43, 14831487 (1995).

2) Kutsuma T., Nagayama I., Okazaki T., Sakamoto T., Akaboshi S., Heterocycles, 8, 397-401 (1977).

3) Summers W. K., Majovski L. V., Marsh G. M., Tachiki K., Kling A., N. Engl. J. Med., 315, 1241-1245 (1986).

4) Yamamoto M., Shimizu M., Harada M., Terai M., Yamaguti T., Kiso To Rinsyo, 19, 4563-4583 (1985).

5) Instruments, general chromatographic methods, and techniques for determination of serum/brain concentrations have been described in the preceding paper. ${ }^{1)}$

6) Knipe A. C., J. Chem. Soc., Perkin Trans. 2, 5, 589-595 (1973).

7) Suzuki H., Imamura K., Kitamura K., Yoshida T., Hanada T., Sakurai Y., Miki H., Kiso To Rinsyo, 19, 223-235 (1985).

8) Bottari F., Macchia B., Gazz. Chim. Ital., 92, 165-173 (1962).

9) Bottari F., Macchia B., Farmaco. Ed. Sc., 19, 1056-1065 (1964).

10) Amami K., Yamamoto Y., Setoguchi M., Nippon Yakurigaku Zasshi, 85, 71-77 (1985).

11) Yamazaki N., Shintani M., Saji Y., Nagawa Y., Yakubutsu, Seishin Kodo, 5, 1-9 (1985). 